\title{
Estructura diamétrica de árboles en potreros de la región Bajo Mixe, Oaxaca
}

\section{Resumen}

\author{
Alfonso Juárez García* \\ Jerónimo Saragos Méndez \\ El Colegio de la Frontera Sur
}

Los potreros arbolados conforman el paisaje común del trópico mexicano, estos influyen sobre la conectividad de los remanentes de selvas; su estructura y composición se basan en árboles dispersos en todo el campo. Se estudiaron dos potreros de la región Bajo Mixe de Oaxaca y se realizó un inventario de todos sus árboles, se les tomó el diámetro a la altura del pecho (DAP) a todos los individuos con DAP $\geq 10 \mathrm{~cm}$ para evaluar la distribución diamétrica de las especies con más de diez individuos. En el potrero El Mirador se encontraron 225 árboles correspondientes a 33 especies, con un área basal total de $36.57 \mathrm{~m}^{2}$. Se analizó la distribución diamétrica de cinco especies, la mayoría de las cuales tuvo una distribución anormal, solo Tabebuia rosea cuenta con una distribución en J invertida. En el potrero El Mamey se censaron 636 individuos representados en 86 especies, con un área basal total de $53.01 \mathrm{~m}^{2}$; se analizaron 16 especies, siendo Zanthoxylum riedelianum, Croton draco, Schizolobium parahyba, Tabebuia rosea, Quararibea funebris, Cordia alliodora y Bursera simaruba las que registraron distribuciones aproximadas a la J invertida. Los resultados indican diferencias en la distribución diamétrica de los árboles en los potreros de estudio, la mayoría de las especies arbóreas son de diámetros mayores (árboles adultos) y son pocas las de diámetros menores (árboles jóvenes o regeneración). Esta situación se asocia al sistema de producción bovina practicado en cada potrero, así como al uso y el manejo anterior al establecimiento del potrero.

\section{Palabras clave}

Pastizales, distribución diamétrica, regeneración natural, especies pioneras.

Recibido: 4/04/205 - Aceptado: 25/05/2015

*Correos electrónicos: aljuarez@ecosur.edu.mx, jesaragos@ecosur.edu.mx 


\title{
Diametric structure of trees in pastures of the Bajo Mixe Region, Oaxaca
}

\author{
Alfonso Juárez García* \\ Jerónimo Saragos Méndez \\ El Colegio de la Frontera Sur
}

\begin{abstract}
Pastures with trees form the common landscape of Mexican tropics, they have an influence upon the connectivity of the remnants of rainforest; their structure and composition is based on dispersed trees throughout the fields. Two pastures from the Bajo Mixe region in Oaxaca, Mexico were studied and an inventory was done considering all trees with a diameter at breast height ( $\mathrm{DBH}) \geq 10 \mathrm{~cm}$ to evaluate the diametrical distribution of species with more than ten individuals. In the Mirador pasture a total of 225 trees were identified corresponding to 33 species, which had a total basal area of $36.57 \mathrm{~m}^{2}$. The diametric distribution of five species was analyzed, of which most had an abnormal distribution and only Tabebuia rosea presents an inverted J distribution. In the Mamey pasture a total of 636 individuals were registered representing 86 species with a total basal area of $53.01 \mathrm{~m}^{2} ; 16$ species were analyzed where Zanthoxylum riedelianum, Croton draco, Schizolobium parahyba, Tabebuia rosea, Quararibea funebris, Cordia alliodora and Bursera simaruba were the ones that presented an approximated inverted $\mathrm{J}$ distributions. The results indicate differences in the diametric distribution of trees in the pastures, most of the trees are of large diameter (mature trees) and few have smaller diameters (saplings or regeneration). These differences are associated with the cattle production system practiced in each pasture and the previous use and management of the area.
\end{abstract}

\section{KeY WORDS}

Pastures, diametric distribution, natural regeneration, pioneer species. 


\section{Introducción}

Los pastizales donde se desarrolla la ganadería bovina, comúnmente conocidos como potreros, han sido clasificados como sistemas silvopastoriles; en ellos se encuentra una gran variedad de árboles, entre los que podemos mencionar los árboles dispersos, las cercas vivas y las cortinas rompevientos, los cuales ofrecen madera, leña, forraje o sombra para el ganado. Como elementos del paisaje, los árboles ayudan a conectar los numerosos fragmentos o parches de selva, actuando como corredores biológicos para el desplazamiento de la fauna dentro y fuera del fragmento, manteniendo el flujo genético y evitando el aislamiento (Harvey et al., 2011). En el ámbito de la regeneración natural, los árboles en potreros son útiles ya que pueden considerarse núcleos de regeneración: crean un microclima, condiciones de humedad y luz favorables para el establecimiento y la supervivencia de plántulas de especies arbóreas (Guevara, Purata y Van der Maarel, 1986). Sin embargo, la permanencia de estos árboles es inestable debido a complicaciones en la regeneración y en la sobrevivencia de las plántulas, atribuidas al manejo del potrero y al impacto del ganado (Esquivel et al., 2009).

El potrero es el sistema agroecológico más extenso y el más reciente de la historia ambiental mexicana, la mezcla de potreros y remanentes de selva ha resultado en un paisaje diverso (Guevara y Laborde, 2012). En México, según estadísticas del INEGI (2013: 744), existen 1129217 unidades de producción de ganadería bovina y 23316942 cabezas de ganado, lo cual demuestra la importancia ambiental y económica de la actividad ganadera para el país. Por consiguiente, estos espacios de producción dominan grandes áreas geográficas, distribuidos en pequeñas unidades de producción catalogadas como potreros por los productores de México.

Aquí hablaremos de árboles en potreros (AP) en referencia tanto a los sistemas silvopastoriles mencionados como a los árboles con diámetro mayor de $10 \mathrm{~cm}$ y altura de $5 \mathrm{~m}$, incluyendo a las palmas.

El estudio de composición y estructura de la comunidad arbórea en los potreros permite conocer el estado ecológico y de conservación de los AP, así como los elementos que indican su regeneración o envejecimiento. Para el análisis de distribución diamétrica se parte del supuesto de la relación entre el tamaño del diámetro y la edad de los árboles. Las clases diamétricas son representadas mediante histogramas de frecuencias que reflejan la estructura demográfica de 
la población de las especies arbóreas. Una distribución de clases diamétricas en forma de L o exponencial negativa (J invertida) con asimetría a la izquierda de árboles menores asegura en el futuro el establecimiento de las poblaciones, ya que ello significa una mayor proporción de juveniles, así como el reclutamiento o regeneración natural de las especies. Por el contrario, una distribución diametral con asimetría a la derecha o negativa es indicio de árboles mayores, lo cual indica un envejecimiento de la vegetación arbórea (Ajbilou, Marañón y Arroyo, 2003; Melo Cruz y Vargas Ríos, 2002).

De acuerdo con lo anterior, el presente trabajo tiene como objetivo analizar la estructura diamétrica de las especies de árboles más abundantes en dos potreros dedicados a la producción bovina con diferentes sistemas de producción, manejo, edad e historia de uso, así como comparar los resultados, para conocer si estos determinan la estructura de los árboles en los potreros.

\section{Materiales y métodos}

El área de estudio corresponde a dos potreros del ejido Constitución Mexicana, perteneciente al municipio de San Juan Mazatlán, en la región Bajo Mixe de Oaxaca, donde los últimos remanentes de vegetación corresponden a selva alta-mediana subperennifolia, con suelos de tipo luvisol (INEGI, 2015). Los tipos de climas que predominan en la región son cálido húmedo y subhúmedo; las temperaturas máximas absolutas están entre $32{ }^{\circ} \mathrm{C}$ y $42^{\circ} \mathrm{C}$, las mínimas anuales van de los $10^{\circ} \mathrm{C}$ a los $18^{\circ} \mathrm{C}$; la precipitación máxima media anual oscila entre 155 y $4000 \mathrm{~mm}$. El ejido colinda con las dos regiones más húmedas del estado, la RH28 (Papaloapan) y la RH29 (Coatzacoalcos). La vegetación y el uso del suelo predominantes son la selva (37.7\% de la superficie total); le sigue el pastizal (25.2\%), y la agricultura (12.6\%) (Santos Espinoza, 2008).

\section{Potreros de estudio}

Se tomaron como objeto de estudio dos potreros representativos de la región, los cuales tienen un uso extensivo y semiintensivo, dedicados a la ganadería extensiva con producción de carne y doble propósito. El potrero El Mirador

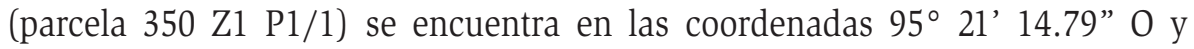
$17^{\circ} 18^{\prime} 35.38^{\prime}$ N, y cuenta con un área de 10 ha. El potrero El Mamey (parcela 
392 Z1 P1/1) se localiza en las coordenadas 95²2’2.67” O” y $17^{\circ} 17^{\prime} 36.99^{\prime \prime} \mathrm{N}$, y tiene una superficie de 18.76 ha. Asimismo, se entrevistó a los propietarios para obtener datos sobre el uso y manejo de los potreros, así como el proceso de cambio de uso de suelo hasta la creación del mismo.

\section{Inventario forestal}

Para el diseño muestral se tomó cada potrero como unidad de muestreo, ya que la investigación sobre AP no implica un patrón homogéneo de distribución y cualquier tipo de parcelas de muestreo tendrá defectos sobre ello (Andrade e Ibrahim, 2003). Se realizó un inventario de todos los árboles con diámetro a la altura del pecho (DAP) $(1.30 \mathrm{~m})$ igual o superior a $10 \mathrm{~cm}$ (siguiendo el supuesto de que si tienen este diámetro ya han superado los filtros ambientales y condiciones de manejo del potrero, además de que el productor ha decidido conservarlos), se registró el nombre común, el DAP y se excluyeron los individuos muertos (Esquivel-Mimenza et al., 2011). Para la identificación de las especies se consultaron las claves de Pennington y Sarukhán (2005: 511), guías y manuales de la vegetación de la región de estudio (Niembro Rocas, Vázquez Torres y Sánchez Sánchez, 2010; Rodríguez Velázquez, Sinaca Colín y Jamangapé García, 2009; Vázquez Torres et al., 2010). A su vez, la nomenclatura de las especies se corroboró en la base de datos Tropicos.org. ${ }^{1}$

Se realizó una estimación de la biomasa arriba del suelo con ecuaciones de Chacón-León y Alice-Harvey (2013: 23) para árboles dispersos en potreros (ecuación 1).

Ecuación 1: Y = 2.9 + 5.05(BA)-0.16(N) + 0.0041 $\left(\mathrm{N}^{2}\right)$

Donde:

$$
\begin{aligned}
& \mathrm{Y}=\text { biomasa } \mathrm{Mg} / \mathrm{ha} \\
& \mathrm{N}=\text { número de árboles/ha } \\
& \mathrm{BA}=\text { área basal } \mathrm{m}^{2} / \mathrm{ha}
\end{aligned}
$$

\section{Estructura diamétrica}

Se analizó mediante la elaboración de histogramas de clases diamétricas con amplitud de $5 \mathrm{~cm}$ para el total de la población observada y para cada especie 
con más de diez individuos. Se utilizaron las tipologías descritas en Ajbilou, Marañón y Arroyo (2003: 114), las recomendaciones de Melo Cruz y Vargas Ríos (2002: 235) y el Coeficiente de Yule (cy) como medida de asimetría de la distribución diametral (ecuación 2).

Ecuación 2: CY $=(\mathrm{X}-\mathrm{Me}) / \mathrm{S}(24)$

Donde:

$\mathrm{X}=$ media

$\mathrm{Me}=$ mediana

$\mathrm{S}=$ desviación estándar

\section{Resultados}

Rancho El Mirador

El origen del potrero es la roza-tumba y quema de la vegetación en 1975 para uso agrícola en cultivos de maíz y arroz, y después el pastoreo de ganado sobre gramas nativas. En 1996 se introdujo pasto insurgente (Brachiaria brizantha). Las razas de ganado son cruza de suizo con cebú, y la carga animal es de 15 cabezas. El sistema de producción es de doble propósito (carne y leche), la leche que se obtiene con ordeña manual y la carne mediante la cría de becerros al destete y el recambio o venta de bovinos para el abasto de carne. La alimentación de los animales es a base de pastos inducidos de tipo insurgente (B. brizantha), gramas nativas y algún tipo de suplementación con alimentos balanceados, silos y sales minerales.

INVENTARIO FORESTAL. Se registraron 225 individuos (22.5 ind/ha-1) representados en 33 especies, las más abundantes son: Sabal mexicana (45), Cedrela odorata (37), Tabebuia rosea (29), Cordia alliodora (17) y Miconia argentea (13) (cuadro 1). Área basal total de $36.57 \mathrm{~m}^{2}$ (3.65 $\left.\mathrm{m}^{2} \mathrm{ha}^{-1}\right)$; biomasa promedio: 17.95 $\mathrm{Mg} / \mathrm{ha}$; diámetro medio de los árboles: $36.32 \mathrm{~cm}$ (desv. tip. 27.43) y área basal media de $0.1621 \mathrm{~m}^{2} \mathrm{ha}^{-1}$ (desv. tip. 0.33).

DistribuCIONES DiAmÉTRICAs. La distribución de la comunidad arbórea es irregular, pero tiende a ser exponencial negativa o en forma de $\mathrm{J}$ invertida (figura 1), aunque no están representadas todas las clases diamétricas. Se analizaron 
cinco especies: T. rosea es la única que tiene una distribución con un patrón en forma de $\mathrm{J}$ invertida, $C$. alliodora y $S$. mexicana presentan distribución asimétrica negativa; $C$. odorata y $M$. argentea evidencian patrones de distribución anormales. Los histogramas de frecuencia y el coeficiente de Yule se muestran en la figura 2.

\section{Rancho El Mamey}

La roza-tumba y quema de la vegetación se realizó en 1997, y permaneció en acahual hasta que se introdujo pasto insurgente (B. brizantha) en 2007. El ganado predominante es criollo de cruzas suizo con cebú, la carga animal es de 25 cabezas; solo se produce carne y se realiza pastoreo extensivo con sistema de rotación en divisiones conocidas como "travesillas". La alimentación se complementa con sales minerales.

INVENTARIO FORESTAL. Se registraron 636 árboles (33.9 ind/ha ${ }^{-1}$ ) distribuidos en 86 especies, las más abundantes fueron S. mexicana (70), C. alliodora (50), S. parahyba (46), T. rosea (38) y S. lateriflorum (37) (cuadro 2). Área basal total de $53.01 \mathrm{~m}^{2}$ (2.83 $\mathrm{m}^{2} \mathrm{ha} \mathrm{H}^{-1}$ ); biomasa promedio: $12.02 \mathrm{Mg} / \mathrm{ha}$; diámetro medio: $27.65 \mathrm{~cm}$ (desv. tip. 17.23), y área basal media de $0.083 \mathrm{~m}^{2}$ (desv. tip. 0.132).

DistRIBUCIONES DIAMÉTRICAS. La distribución diamétrica de la comunidad arbórea está representada en forma de J invertida (figura 3). Se analizaron 16 especies, las distribuciones diamétricas encontradas y las especies que la presentan son: asimétrica positiva: S. lateriflorum, T. rosea, $R$. menbranacea, $Q$. funebris, C. alliodora, B. simaruba, A. graveolens y S. mombin; asimétrica negativa: P. sapota; exponencial negativa: Z. riedelianum, C. draco y S. parahyba; distribución normal: $S$. mexicana y $C$. brasiliense; distribución anormal: A. inermis y B. alicastrum. Las distribuciones diamétricas de las especies analizadas se pueden constatar en la figura 4.

\section{Discusión}

De acuerdo con las tendencias de distribución diamétrica de las especies analizadas, se encontraron seis especies que presentan ventajas de sobrevivencia o regeneración en el rancho El Mamey: Z. riedelianum, C. draco, S. parahyba, 
T. rosea, Q. funebris, C. alliodora y B. simaruba (figura 4). En el rancho El Mirador, T. rosea fue la única especie que presentó esta situación, como se aprecia en la figura 2. Estas especies tienen patrones próximos a distribuciones de forma exponencial negativa (J invertida) y asimétricas positivas, lo cual asegura la existencia de individuos jóvenes que a la larga reemplazarán a los adultos y, por ende, su existencia en los potreros (Ajbilou, Marañón y Arroyo, 2003; Imaña Encinas, Antunes Santana y Rainier Imaña, 2011; Restrepo, Orrego y Galeano, 2012; Vargas et al., 2010). Este patrón coincide con especies pioneras de hábito heliófito demandantes de luz, que son comunes en etapas de sucesión temprana o en áreas perturbadas y que además toleran las condiciones de micrositio de las pasturas, el ramoneo y pisoteo del ganado (Esquivel et al., 2009; Román Dañobeytia et al., 2007), lo que sugiere que solo necesitan unos cuantos meses de descanso del potrero para regenerarse y establecerse.

La distribución diamétrica con asimetría negativa y de tipo normal representa casos donde la mayoría de los individuos son adultos; esto puede originarse cuando árboles de mayor edad se dejan en pie durante el proceso de roza-tumba y logran sobrevivir al proceso de quema (Van der Wal, Espinoza-López, y Dzib-Castillo, 2011), lo que a su vez repercute en y compromete la regeneración de la especie en el potrero (Imaña Encinas, Antunes Santana y Rainier Imaña, 2011). Las especies en esta situación en el rancho El Mamey son P. sapota, S. mexicana y $C$. brasiliense (figura 4); en el rancho El Mirador, C. alliodora y $S$. mexicana. Estas especies poseen condiciones de regeneración limitada, como evidencian los resultados de Román Dañobeytia et al. (2007: 8), que establecen que especies umbrófilas como $C$. brasiliense tienen niveles de supervivencia y establecimientos inferiores respecto de especies heliófitas. Este patrón de distribución indica la presencia de árboles incapaces de reproducirse y regenerarse en las condiciones de los potreros. Esquivel et al. (2009: 83) se refiere a este tipo de árboles como individuos living deads, de los cuales solo se espera su desaparición en el potrero.

Por otro lado, los patrones de distribución diamétrica anormal, como en el caso de A. inermis y B. alicastrum en el potrero El Mamey (figura 4), y C. odorata y $M$. argentea en El Mirador (figura 2) sugieren eventos de tala o aprovechamientos selectivos de individuos por parte del productor, como señalan Imaña Encinas, Antunes Santana y Rainier Imaña (2011: 28). Este 
aprovechamiento puede observarse en especies maderables (de manera señalada el cedro), las cuales una vez que alcanzan diámetros comerciales son cortadas y vendidas por el productor, lo que explica los claros o huecos en las gráficas de distribución.

La caracterización de ambos potreros resultado de esta investigación indica que la historia, edad, sistema de producción y manejo de cada potrero influyen sobre la densidad, la diversidad y la distribución diamétrica de los individuos en los potreros. Villacís (2008:12) menciona que en potreros con sistemas de carne y mixto la densidad de árboles es casi el doble que en sistemas de leche y doble propósito. Esto es así porque los productores con sistema de leche y doble propósito no toleran los árboles por el efecto negativo de la sombra sobre la producción de pastos, y porque es común la corta-venta de las especies maderables para obtener ingresos adicionales.

En el rancho El Mirador, donde el sistema de producción es doble propósito, predominan especies arbóreas maderables como C. odorata, T. rosea, C. alliodora y S. mexicana (cuadro 1). El diámetro promedio de los árboles suele ser mayor, por consiguiente, son árboles de mayor tamaño y biomasa (Villacís, 2008), como consecuencia del manejo realizado por los productores. En El Mirador, que tiene mayor antigüedad y uso ganadero, los árboles presentes son remanentes de selva (que se encuentra en fase senil), y los árboles de menor edad se ven afectados a causa de la intolerancia a más árboles (pues más cantidad de sombra menoscaba el desarrollo de los pastos) en el potrero, además de la incidencia de actividades de manejo como el chapeo, uso de herbicidas, ramoneo y pisoteo del ganado. La distribución diamétrica de los árboles indica que en sistemas intensificados de producción como en El Mirador la mayoría de los árboles presentan distribuciones asimétricas negativas y anormales, pero esto no asegura su permanencia en los potreros por la falta de individuos jóvenes. Solo T. rosea revela una distribución exponencial negativa que asegura la permanencia de la especie. Cabe mencionar que este árbol es considerado por los productores como una especie de maleza por ser abundante en etapas juveniles, así que es necesario el chapeo y dejar solo algunos.

En comparación con el sistema productivo carne o mixto (rancho El Mamey), donde el manejo es menos intensivo, los árboles tienden a ocupar las distintas categorías diamétricas; en este caso el potrero es de reciente creación 
y hay mayor diversidad de especies cuyo origen es el proceso de roza-tumba y quema de la selva durante su creación. En este rancho hay individuos adultos y juveniles, resultado de la regeneración de las especies que superan los filtros del ambiente y el manejo del potrero, lo que provoca que haya cantidades de biomasa menores que en el otro sistema de manejo, pues los árboles son de menores diámetros y edad. Además, en el potrero El Mamey hay un posible efecto de las travesillas, que permiten la rotación del ganado por un lapso aproximado de un mes, lo cual da tiempo suficiente para el establecimiento de especies heliófitas o pioneras. En este potrero y sistema de producción la mayoría de las especies analizadas presentan distribuciones diamétricas positivas y exponencial negativa, lo que les confiere persistencia; solo tres especies $(P$. sapota, S. mexicana y $C$. brasiliense ofrecen escenarios negativos por contar mayormente con árboles adultos.

La especie C. alliodora, común en ambos sistemas de producción, es un claro ejemplo de diferenciación entre ambos potreros. En El Mirador la distribución diamétrica de esta especie es asimétrica negativa, y su tendencia de desaparición en el corto plazo es latente. Mientras que en El Mamey la distribución es asimétrica positiva, por lo que puede asegurar su regeneración y sobrevivencia.

Las especies que presentan distribución diamétrica en J invertida, que evidencian regeneración abundante en los potreros, pueden ser aprovechadas mediante manejo silvícola: especies maderables como el picho (S. parahyba), tachuelillo ( $Z$. riedelianum), gateado (A. graveolens) y el roble (T. rosea).

La tendencia observada del uso y manejo de los Ap indica que, con el tiempo, la selectividad de los árboles por parte del productor lleva a una considerable reducción de la diversidad de especies, ya que solo se permite el crecimiento de árboles que ofrezcan beneficios maderables, como cerco vivo, frutas, forrajeras y sombra para el ganado (Harvey et al., 2011).

Los AP representan un gran potencial para incrementar y mantener reservas de carbono que ayuden a mitigar el cambio climático, por lo cual es de suma importancia su fomento y reconocimiento por parte de los productores y de las instituciones que fungen como promotores de la conservación y manejo de los recursos forestales (Chacón-León y Alice-Harvey, 2013). En este sentido, es necesario conocer las implicaciones del establecimiento de sistemas silvopastoriles con árboles en los potreros e investigar las causas de la limitación de 
la regeneración de las especies para contribuir a la conservación de árboles a largo plazo (López et al., 2007; Useche, Harvey y Declerck, 2011; Villacís, 2008; Villanueva et al., 2003; Zapata et al., 2006). Para el logro de estos objetivos será necesario diseñar e implementar políticas que apoyen la conservación, como el pago por servicios ambientales (Zapata et al., 2006), además de tener en consideración el sistema de producción y manejo que los productores hacen de los potreros.

\section{Conclusiones}

La estructura diamétrica de las especies analizadas es diferente en los dos casos de estudio, ello indica que la historia, edad, sistema de producción y manejo de los potreros influyen sobre la densidad, diversidad, biomasa y distribución diamétrica de los árboles. Sin embargo, el sistema de rotación del ganado en el rancho El Mamey mediante el uso tradicional de travesillas, aunado a la poca intensidad de utilización del área antes de ser convertido a potrero, puede asociarse a la regeneración positiva de especies arbóreas y a la abundancia de las mismas.

En ambos potreros la mayoría de los árboles son adultos y solo unas especies cuentan con árboles jóvenes, que después de algunos años dejarán de existir sin ser reemplazados. Las especies con mejores posibilidades de persistir (por tener mayor abundancia e individuos jóvenes) son T. rosea, S. parahyba, S. lateriflorum, C. draco y Z. riedelianum. A su vez, las especies que desaparecerán por falta de juveniles en el rancho El Mirador son C. alliodora y S. mexicana; en el rancho El Mamey, S. mexicana, C. brasiliense, P. sapota y B. alicastrum. Esto implica, además, la desaparición de otras especies de plantas y animales asociados a su sobrevivencia o hábitat. En consecuencia, la biodiversidad en los potreros aún arbolados es amenazada por la reducción de árboles y por diversos elementos de manejo, así como por la edad y el uso que se les da. De ahí la trascendencia de fomentar la inclusión de árboles en los potreros teniendo en cuenta los factores y elementos descritos en este trabajo. 


\section{Agradecimientos}

Agradecemos al Dr. Pablo Martínez Zurimendi por el apoyo metodológico y sugerencias de mejora a este escrito, así como el apoyo recibido del proyecto a su cargo "Diagnóstico del estado silvícola y desarrollo de plan de manejo en plantaciones de teca (Tectona grandis L.f.) y melina (Gmelina arborea Roxb.) en Tabasco" financiado por Fomix Tabasco. A los productores que facilitaron el área de estudio para la investigación.

\section{Fuentes consultadas}

Ajbilou, R., T. Marañón y J. Arroyo (2003). “Distribución de clases diamétricas y conservación de bosques en el norte de Marruecos”. Investigación Agraria. Sistema y Recursos Forestales, 12 (2), 111-123.

Andrade, H. J. y M. Ibrahim (2003). “¿Cómo monitorear el secuestro de carbono en los sistemas silvopastoriles?” Agroforestería en las Américas, 10 (39-40), 109-116.

Chacón-León, M. y C. A. Harvey (2013). "Reservas de biomasa de árboles dispersos en potreros y mitigación al cambio climático". Agronomía Mesoamericana, 24 (1), 17-26.

Esquivel, M. J. et al. (2009). "Regeneración natural de árboles y arbustos en potreros activos de Nicaragua”. Agroforestería en las Américas, 47, 76-84.

Esquivel-Mimenza, H. et al. (2011). "Dispersed Trees in Pasturelands of Cattle Farms in a Tropical Dry Ecosystem". Tropical and Subtropical Agroecosystems, 14 (3), 933-941.

Guevara, S. y J. Laborde (2012). “The Mesoamerican Rain Forest Environmental History. Livestock and Landscape Biodiversity at los Tuxtlas, México”. Pastos. Revista de la Sociedad Española para el Estudio de los Pastos, 42 (2), 219-248.

, S. E. Purata y E. Van der Maarel (1986). "The Role of Remnant Forest Trees in Tropical Secondary Succession”. Vegetatio, 66, 77-84.

Harvey, C. A. et al. (2011). "Conservation Value of Dispersed Tree Cover Threatened by Pasture Management”. Forest Ecology and Management, 261 (10), 1664-1674.

Imaña Encinas, J., O. Antunes Santana y C. Rainier Imaña (2011). “Estructura diamétrica de un fragmento del bosque tropical seco de la región del Eco-museo del Cerrado, Brasil”. Colombia Forestal, 14 (1), 23-30. 
INEGI (2013). Anuario estadístico y geográfico por entidad federativa 2013. México: Instituto Nacional de Estadística y Geografía.

(2015). “Mapa Digital de México” [en línea]. México: Instituto Nacional de Estadística y Geografía. Disponible en: http://gaia.inegi.org.mx/ mdm6/

López, F. et al. (2006). “Toma de decisiones de productores ganaderos sobre el manejo de los árboles en potreros en Matiguás, Nicaragua”. Agroforestería en las Américas, 45, 93-99.

Melo Cruz, O. A. y R. Vargas Ríos (2002). Evaluación ecológica y silvicultural de ecosistemas boscosos. Ibagué: Universidad del Tolima.

Niembro Rocas, A., M. Vázquez Torres y O. Sánchez Sánchez (2010). Árboles de Veracruz. 100 especies para la reforestación estratégica. México: Gobierno del Estado de Veracruz/Secretaría de Educación del Estado de Veracruz-Comisión del Estado de Veracruz de Ignacio de la Llave para la Conmemoración de la Independencia Nacional y la Revolución/Centro de Investigaciones Tropicales.

Pennington, T. D. y J. Sarukhán (2005). Árboles tropicales de México. Manual para la identificación de las principales especies. 3a ed. México: Universidad Nacional Autónoma de México/Fondo de Cultura Económica.

Restrepo, H. I., S. A. Orrego y O. J. Galeano (2012). "Estructura de bosques secundarios y rastrojos montano bajos del norte de Antioquia, Colombia”. Colombia Forestal, 15 (2), 173-189.

Rodríguez Velázquez, J., P. Sinaca Colín y G. Jamangapé García (2009). Frutos y semillas de árboles tropicales de México. México: Secretaría de Medio Ambiente y Recursos Naturales/Instituto Nacional de Ecología.

Román Dañobeytia, F. et al. (2007). "Establecimiento de seis especies arbóreas nativas en un pastizal degradado en la Selva Lacandona, Chiapas, México”. Ecología Aplicada, 6 (1-2), 1-8.

Santos Espinoza, J. (2008). "Resumen Ejecutivo. Estudio regional forestal. Unidad de Manejo Forestal Bajo Mixe [en línea]. Oaxaca: Unidad de Manejo Forestal del Bajo Mixe. Disponible en: http://www.conafor.gob. mx:8080/documentos/docs/9/1099ERF_UMAFOR2014.pdf

Useche, D., Harvey, C. A., y Declerck, F. (2011). Implicaciones sociales, económicas y ecológicas para la implementación de sistemas silvopastoriles 
como estrategia para la conservación de la biodiversidad en paisajes ganaderos tropicales. Agroforestería en las Américas, (48), 84-93.

Van der Wal, J. C., Espinoza-López, R., y Dzib-Castillo, B. (2011). Evaluación preliminar del desmonte selectivo para establecer potreros diversificados en San José Carpizo No. 1, Campeche, México. Universidad y Ciencia, 27(1), 219-226.

Vargas, R. et al. (2010). "Spatial Distribution and Regeneration Strategies of the Main Forest Species on Robinson Crusoe Island". Revista Chilena de Historia Natural, 83 (3), 349-363.

Villacís, J. (2008). Contribución de los árboles dispersos en potreros a los sistemas de producción ganadera en Rio Frio, Costa Rica. Agroforestería Ecológica, 1-12. Recuperado de: www.agroforesteriaecologica.com Villanueva, C., Ibrahim, M., Harvey, C. A., Sinclair, F. L., y Muñoz, D. (2003). Decisiones claves que influyen sobre la cobertura arbórea en fincas ganaderas de Cañas, Costa Rica. Agroforestería en las Américas, 10(3940), 69-77.

Vázquez Torres, M., Armenta Montero, S., Campos Jiménez, J., \& Carvajal Hernández, C. I. (2010). Árboles de la región de Los Tuxtlas. México: Gobierno del Estado de Veracruz, Secretaría de Educación del Estado de Veracruz, Comisión del Estado de Veracruz de Ignacio de la Llave para la Conmemoración del Bicentenario de la Independencia Nacional y del Centenario de la Revolución.

Zapata, Á., Murgueitio, E., Mejía, C., Zuluaga, A. F., y Ibrahim, M. (2006). Efecto del pago por servicios ambientales en la adopción de sistemas silvopastoriles en paisajes ganaderos de la cuenca media del río La Vieja, Colombia. Agroforestería en las Américas, (45), 86-92. 


\section{Apéndice}

Cuadro 1. Principales especies de árboles dispersos en el potrero del rancho El Mirador

\begin{tabular}{|c|c|c|c|c|}
\hline NOMBRE COMÚN & NOMBRE CIENTífICO & FAmilia & ABUndANCIA & $\begin{array}{c}\text { ÁREA } \\
\text { BASAL M }\end{array}$ \\
\hline Palma & Sabal mexicana Mart., 1839 & Palmae & 45 & 1.58 \\
\hline Cedro & Cedrela odorata L., 1759 & Meliaceae & 37 & 4.91 \\
\hline Roble & Tabebuia rosea (Bertol.) DC., 1845 & Bignoniaceae & 29 & 1.34 \\
\hline Solerilla & $\begin{array}{l}\text { Cordia alliodora (Ruiz \& Pav) Oken, } \\
1841\end{array}$ & Boraginaceae & 17 & 2.18 \\
\hline Capulín & Miconia argentea (Sw.) DC., 1828 & Melastomataceae & 13 & 1.84 \\
\hline Anonilla & $\begin{array}{l}\text { Rollinia menbranacea Triana \& } \\
\text { Planch, } 1862\end{array}$ & Annonaceae & 8 & 0.88 \\
\hline Tachuelillo & Zanthoxylum riedelianum Engl., 1878 & Rutaceae & 8 & 0.57 \\
\hline Aguacate & Persea americana Mill., 1768 & Lauraceae & 7 & 0.71 \\
\hline Guanacastle & $\begin{array}{l}\text { Enterolobium cyclocarpum (Jacq.) } \\
\text { Griseb., } 1860\end{array}$ & Mimosoideae & 7 & 7.39 \\
\hline Cocoite & Gliricidia sepium (Jacq.) Steud., 1842 & Faboideae & 5 & 0.13 \\
\hline Amate & Ficus cotinifolia Kunth, 1817 & Moraceae & 4 & 5.30 \\
\hline Mulato & Bursera simaruba (L) Sarg., 1890 & Burseraceae & 4 & 0.65 \\
\hline Tololote & $\begin{array}{l}\text { Andira inermis (W. Wright) DC., } \\
1825\end{array}$ & Faboideae & 4 & 0.78 \\
\hline Ceiba & Ceiba pentandra (L) Gaertn, 1791 & Bombacaceae & 3 & 2.54 \\
\hline Jabín & Piscidia piscipula (L) Sarg., 1891 & Faboideae & 3 & 0.31 \\
\hline Jobo & Spondias mombin L., 1753 & Anacardiaceae & 3 & 0.89 \\
\hline Limón & Citrus limonia (L.) Osbeck, 1765 & Rutaceae & 3 & 0.07 \\
\hline Picho & $\begin{array}{l}\text { Schizolobium parahyba (Vell.) Blake, } \\
1919\end{array}$ & Caesalpinioideae & 3 & 0.21 \\
\hline Tres lomos & $\begin{array}{l}\text { Cupania dentata Moc. \& Sessé ex DC., } \\
1824\end{array}$ & Sapindaceae & 3 & 0.42 \\
\hline Tulipán & $\begin{array}{l}\text { Spathodea campanulata P. Beauv, } \\
1805\end{array}$ & Bignoniaceae & 3 & 0.67 \\
\hline \multicolumn{3}{|c|}{ Otras especies (13) } & 16 & 3.21 \\
\hline \multicolumn{3}{|l|}{ Total } & 225 & 36.57 \\
\hline
\end{tabular}

Fuente: Elaboración propia. 
Cuadro 2. Principales especies de árboles dispersos en el potrero del rancho El Mamey

\begin{tabular}{|c|c|c|c|c|}
\hline $\begin{array}{l}\text { NOMBRE } \\
\text { COMÚN }\end{array}$ & NoMBRE CIENTíFICO & FAMILIA & ABUNDANCIA & $\begin{array}{c}\text { ÁREA } \\
\text { BASAL M }{ }^{2}\end{array}$ \\
\hline Palma & Sabal mexicana Mart., 1839 & Palmae & 70 & 2.44 \\
\hline Solerilla & $\begin{array}{l}\text { Cordia alliodora (Ruiz \& Pav) Oken, } \\
1841\end{array}$ & Boraginaceae & 50 & 1.80 \\
\hline Picho & $\begin{array}{l}\text { Schizolobium parahyba (Vell.) Blake, } \\
1919\end{array}$ & Caesalpinioideae & 46 & 1.86 \\
\hline Roble & Tabebuia rosea (Bertol.) DC. 1845 & Bignoniaceae & 38 & 1.00 \\
\hline Chilamate & Sapium lateriflorum Hemsl., 1901 & Euphorbiaceae & 37 & 1.12 \\
\hline Jobo & Spondias mombin L., 1753 & Anacardiaceae & 36 & 6.60 \\
\hline Drago & Croton draco Schltdl. \& Cham., 1831 & Euphorbiacea & 29 & 0.46 \\
\hline Tololote & Andira inermis (W. Wright) DC., 1825 & Faboideae & 26 & 2.78 \\
\hline Anonilla & $\begin{array}{l}\text { Rollinia menbranacea Triana \& } \\
\text { Planch, } 1862\end{array}$ & Annonaceae & 25 & 1.02 \\
\hline Mulato & Bursera simaruba (L) Sarg., 1890 & Burseraceae & 20 & 1.96 \\
\hline Ojoche & Brosimun alicastrum Sw., 1788 & Moraceae & 19 & 4.86 \\
\hline Gateado & Astronium graveolens Jacq., 1760 & Anacardiaceae & 16 & 0.84 \\
\hline Mamey & $\begin{array}{l}\text { Pouteria sapota (Jacq.) H. E. Moore \& } \\
\text { Stearn } 1967\end{array}$ & Sapotaceae & 16 & 3.05 \\
\hline Tachuelillo & Zanthoxylum riedelianum Engl., 1874 & Rutaceae & 16 & 0.45 \\
\hline Molinillo & $\begin{array}{l}\text { Quararibea funebris (La Llave) } \\
\text { Vischer, } 1919\end{array}$ & Bombacaceae & 14 & 0.80 \\
\hline Lechemaria & $\begin{array}{l}\text { Calophyllum brasiliense Cambess, } \\
1825\end{array}$ & Guttiferae & 10 & 1.32 \\
\hline Mala mujer & Alchornea latifolia Swarts., 1788 & Euphorbiacea & 9 & 1.63 \\
\hline Cedro & Cedrela odorata L., 1759 & Meliaceae & 8 & 0.88 \\
\hline Ceiba & Ceiba pentandra (L) Gaertn 1791 & Bombacaceae & 7 & 4.98 \\
\hline Guásimo & Guazuma ulmifolia Lam 1789 & Sterculiaceae & 7 & 0.82 \\
\hline Majagua & $\begin{array}{l}\text { Robinsonella mirandae Gómez Pompa } \\
1962\end{array}$ & Malvaceae & 6 & 0.64 \\
\hline Golondrina & $\begin{array}{l}\text { Pleuranthodendron lindenii (Turcz.) } \\
\text { Sleumer } 1978\end{array}$ & Tiliaceae & 5 & 0.19 \\
\hline \multicolumn{3}{|c|}{ Otras especies (64) } & 126 & 11.51 \\
\hline \multicolumn{3}{|l|}{ Total } & 636 & 53.01 \\
\hline
\end{tabular}

Fuente: Elaboración propia. 


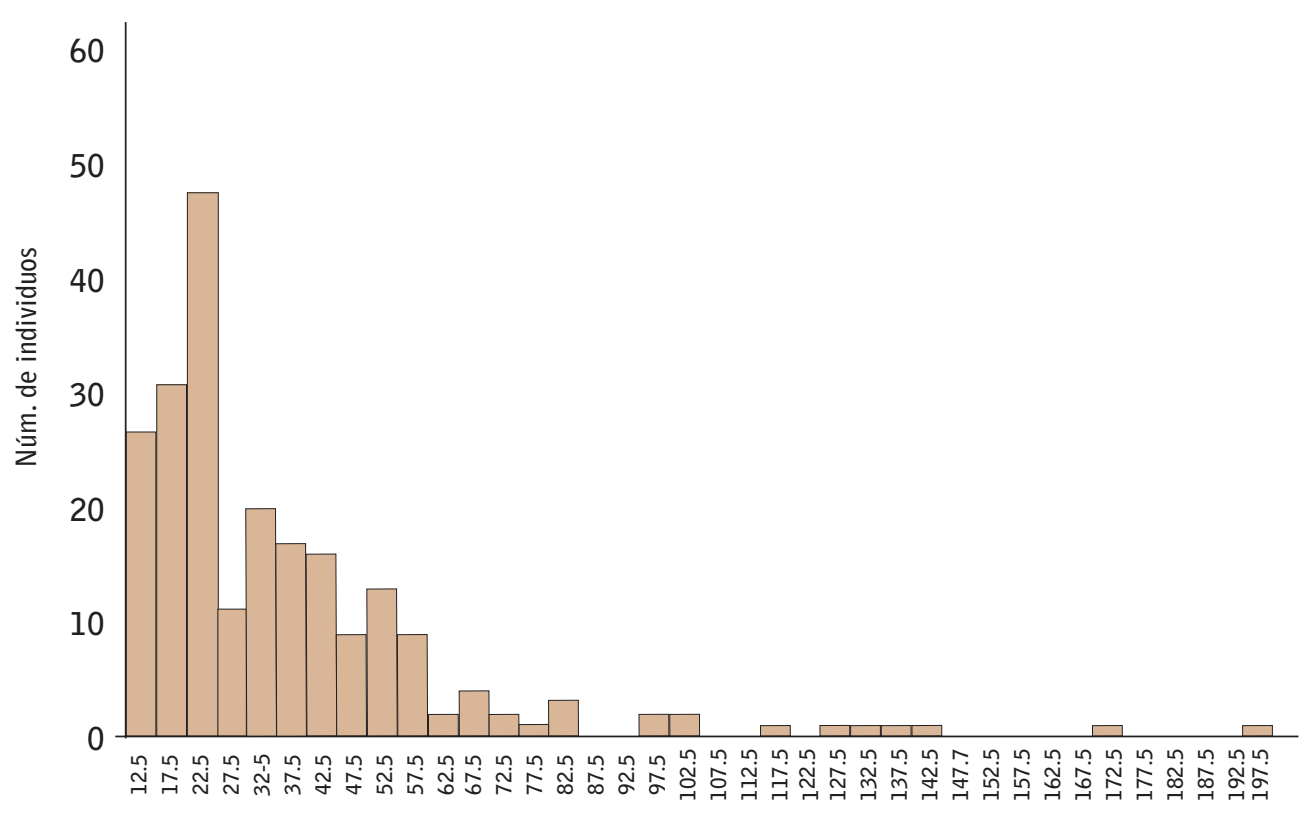

Marcas de clase diamétrica

Figura 1. Distribución diamétrica de la comunidad arbórea del potrero El Mirador 

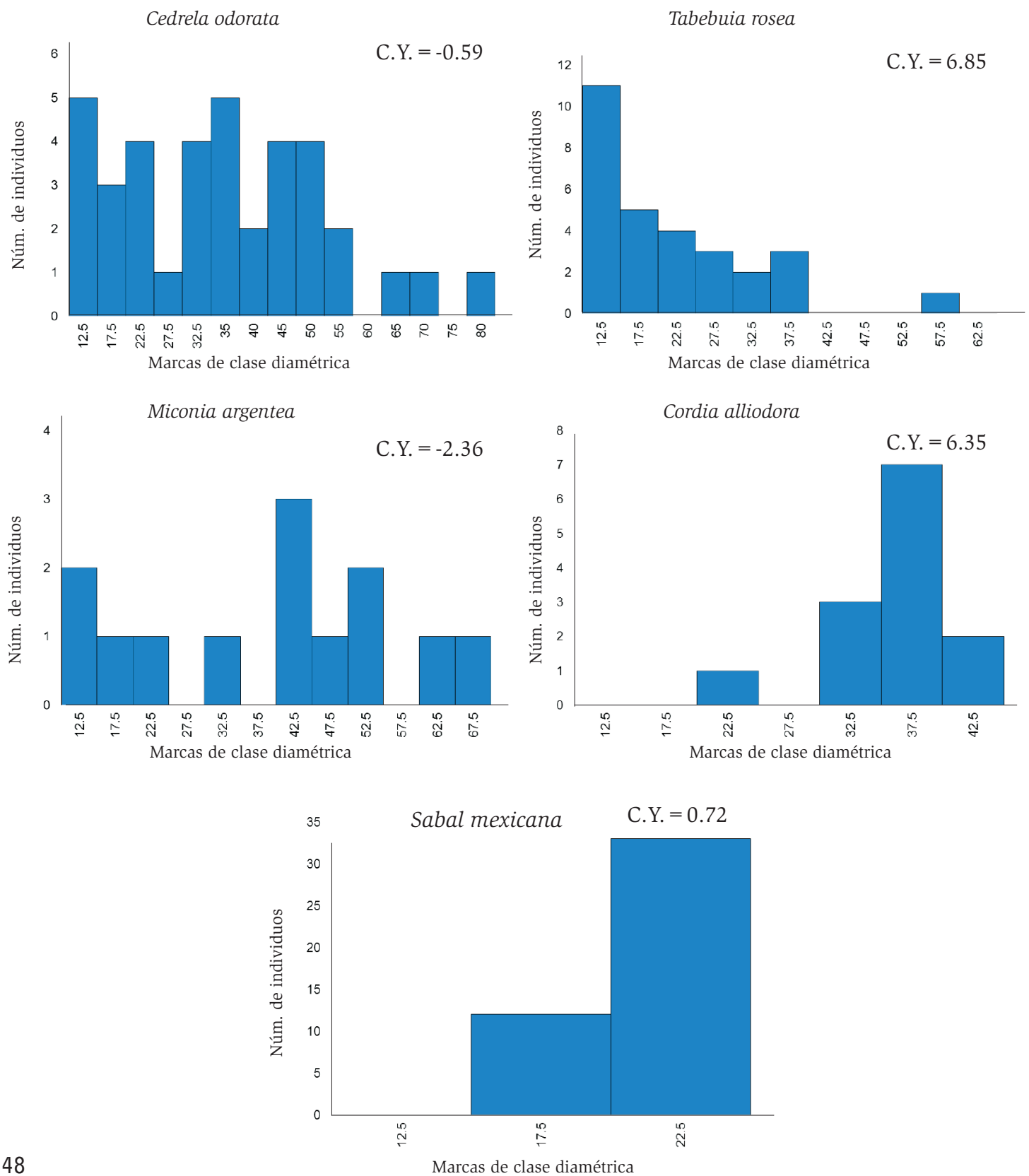

Figura 2. Patrones de distribución diamétrica y coeficiente de Yule de las especies del rancho El Mirador 


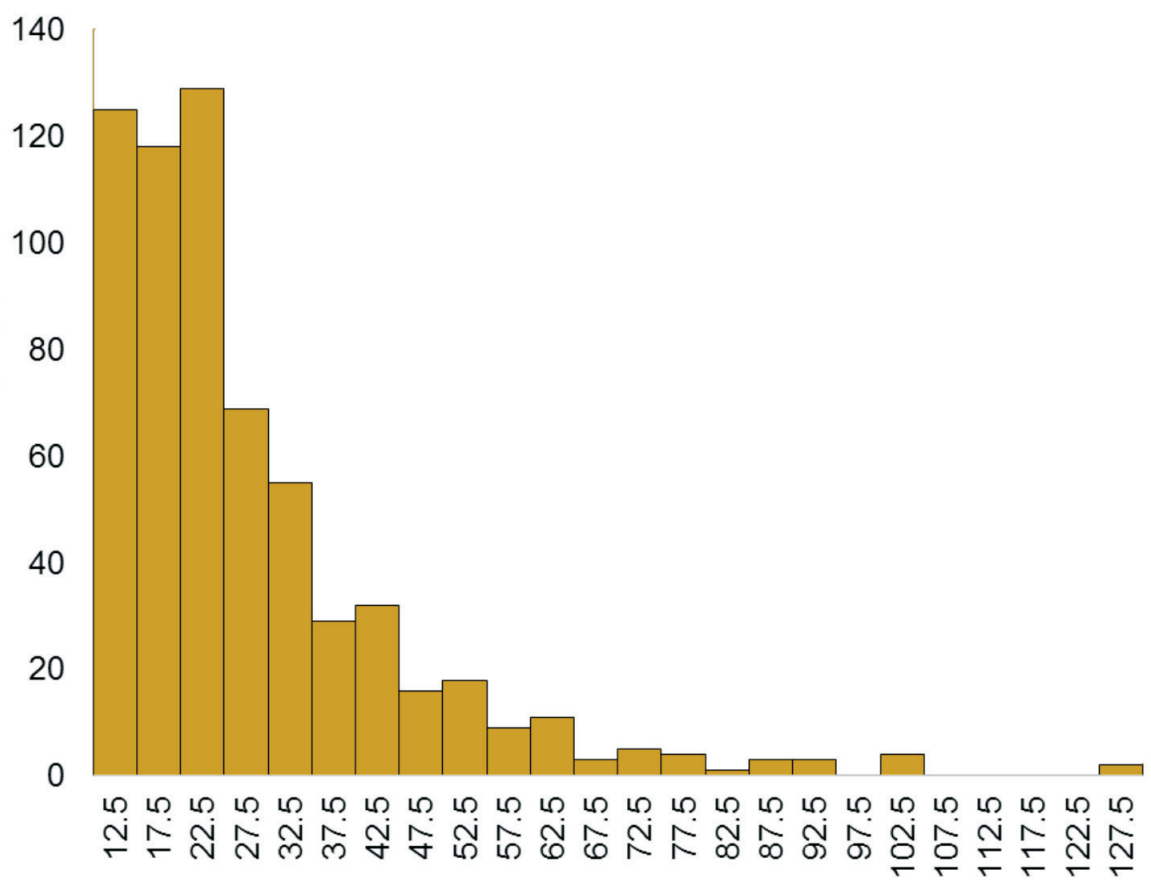

Figura 3. Distribución diamétrica de la comunidad arbórea del potrero El Mamey 
Estructura diamétrica de árboles en potreros de la región Bajo Mixe, Oaxaca

Sapium lateriflorum

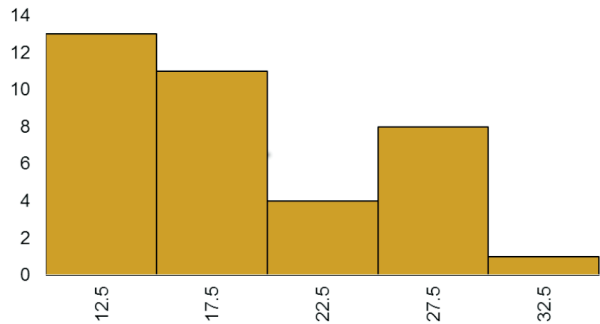

Tabebuia rosea

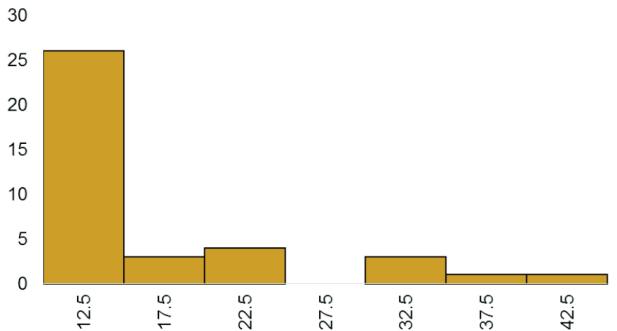

Rollinia menbranacea

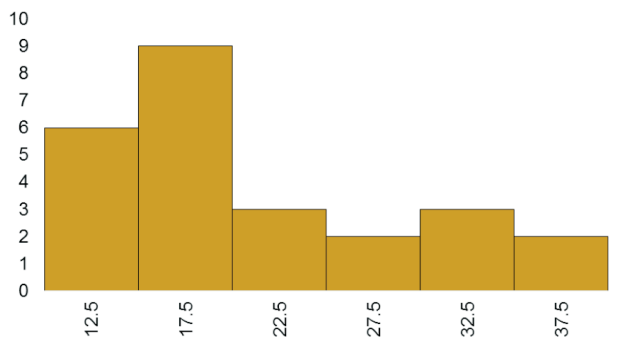

Cordia alliodora

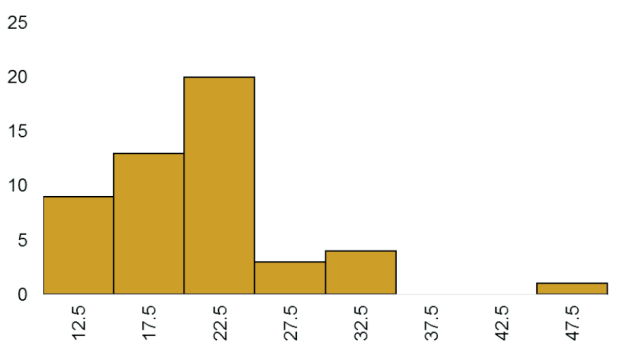

Zanthoxylum riedelianum

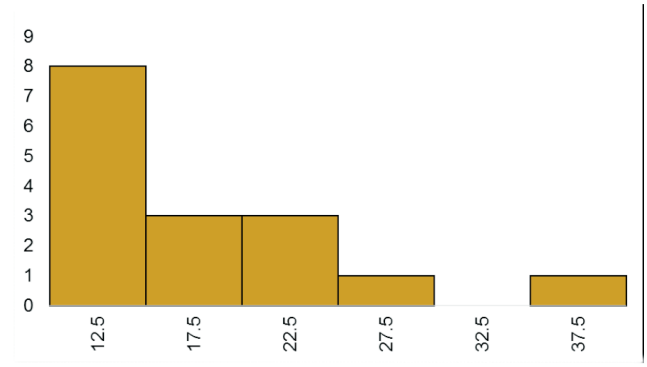

Croton draco

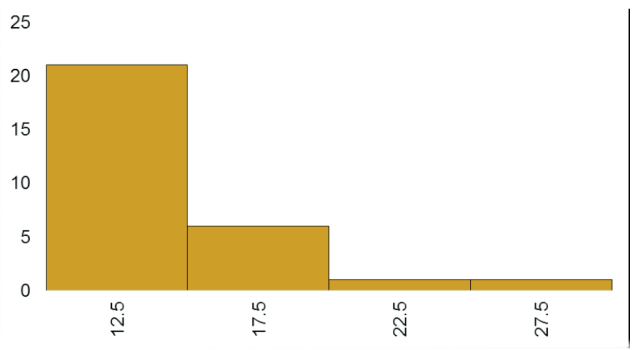

Quararibea funebris

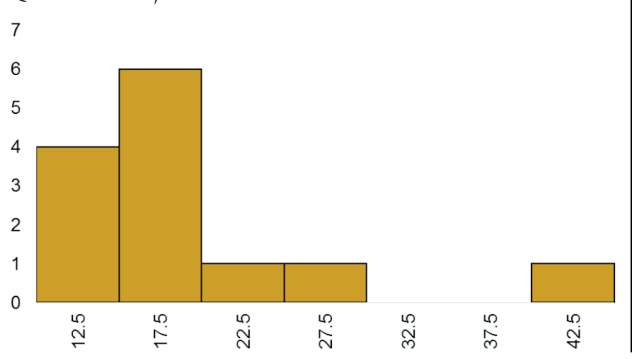

Sabal mexicana

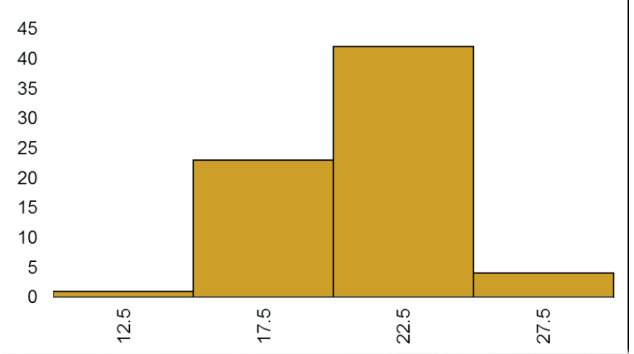

Figura 4. Patrones de distribución diamétrica y coeficiente de Yule de las especies del rancho El Mamey (continúa) 
${ }_{5}$ Brusera simaruba

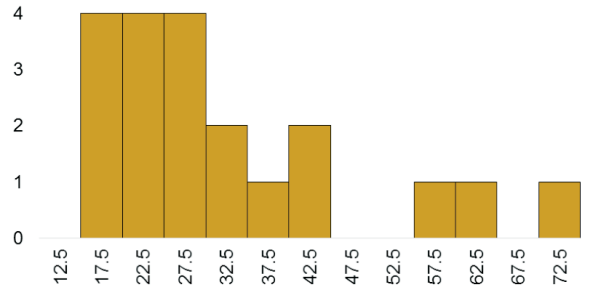

Schizolobium parahyba

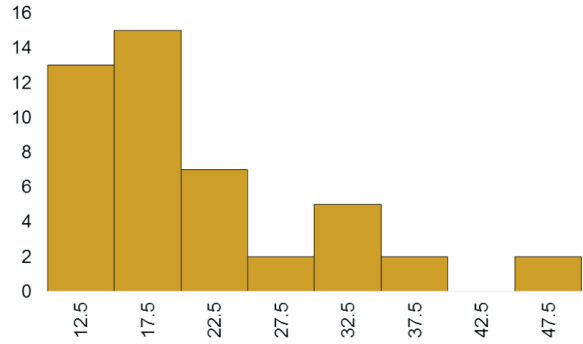

Astronium graveolens

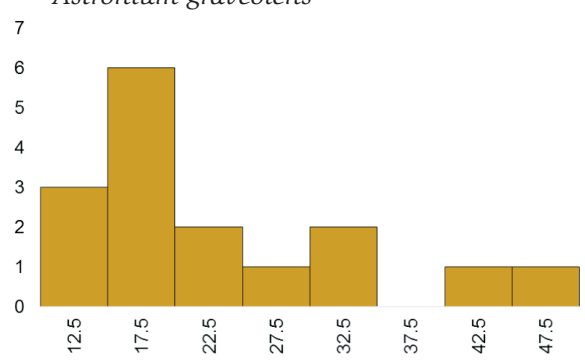

Brosimun alicastrum

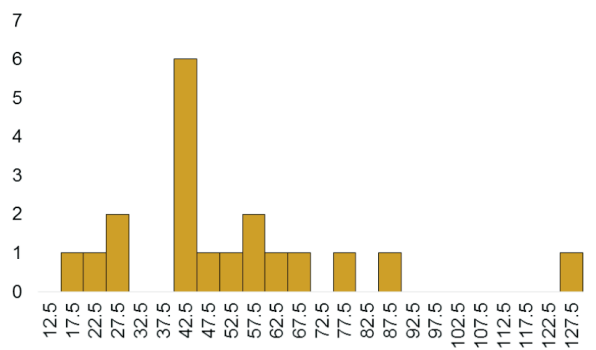

Andira inermis

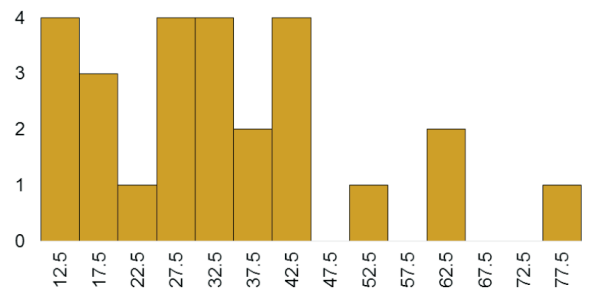

Calophyllum brasiliense

3

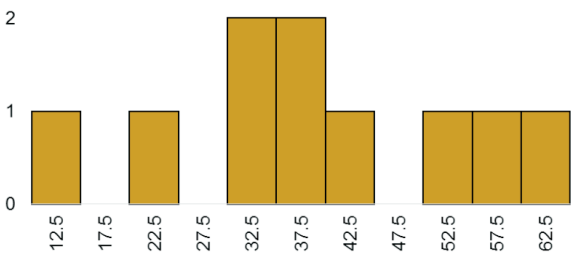

Pouteria sapota

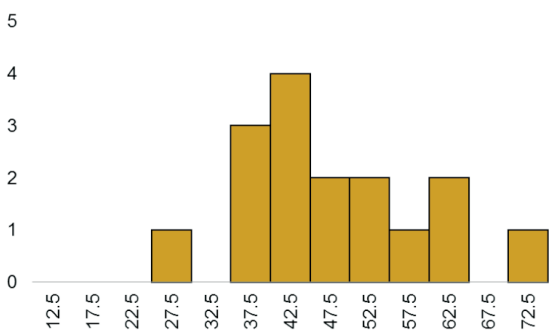

Spondias mombin

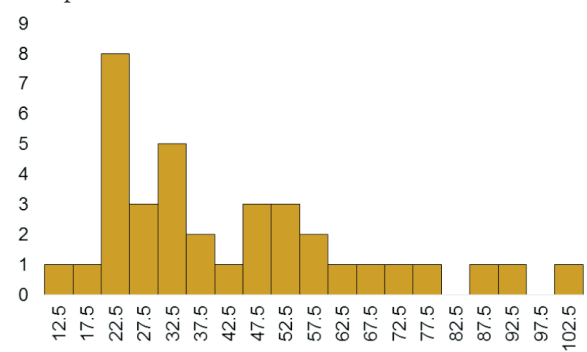

Figura 4. Patrones de distribución diamétrica y coeficiente de Yule de las especies del rancho El Mamey (finaliza) 
